\title{
Enunciación
}

http://revistas.udistrital.edu.co/ojs/index.php/enunc

\section{Pedagogías emergentes en contextos cambiantes: pedagogías en red en la sociedad del conocimiento}

\author{
Emerging pedagogies in changing contexts: Pedagogies in networked \\ knowledge society
}

\author{
Binod Gurung ${ }^{1}$
}

Gurung, B. (2013). Emerging Pedagogies in Changing Contexts: Pedagogies in Networked Knowledge Society. JOGLTEP 1(2), 105-124.

Para citar este artículo: Gurung, B. (2015). Pedagogías emergentes en contextos cambiantes: pedagogías en red en la sociedad el conocimiento. Enunciación, 20(2), pp. 271-286.

Traductora: Amparo Clavijo Olarte, Ph.D.

Doctorado en Educación de la Universidad de Arizona. Docente de la Universidad Distrital Francisco José de Caldas. Correo electrónico: aclavijoolarte@gmail.com

\section{Resumen}

La noción de pedagogías es un concepto multidimensional emergente construido a partir de la relación entre los componentes pedagógicos (como la tecnología, la pedagogía y el contenido) y los contextos pedagógicos cambiantes de la sociedad, la economía, la educación y la ciencia y tecnología para mencionar algunos. Desafortunadamente, para los marcos teóricos tradicionales existentes no es importante la relación entre los componentes y los contextos pedagógicos cambiantes. Las pedagogías tradicionales existentes se ven como estáticas y unidimensionales y se enfocan exclusivamente en los componentes o en los contextos. En este artículo, propongo repensar las pedagogías tradicionales existentes y enmarcarlas como pedagogías emergentes, con el fin de encontrar la naturaleza emergente y multidimensional de la pedagogía en los actuales contextos pedagógicos cambiantes que evolucionan dentro de redes en la sociedad del conocimiento, la economía del conocimiento, la democracia orientada en la diversidad y la alfabetización digital. Así, a través del reconocimiento de las fortalezas y debilidades de dos marcos pedagógicos esenciales -como son el conocimiento pedagógico y tecnológico del contenido (TPCK, por su sigla en inglés), y la educación multicultural (MCE, por su sigla en inglés)-, se plantea una discusión teórica sobre las pedagogías emergentes como concepto y como práctica. Igualmente, se hace una recomendación para futuras investigaciones y teorías relacionadas con las pedagogías emergentes.

Palabras clave: pedagogías emergentes, sociedad del conocimiento en red, economía del conocimiento, democracia orientada en la diversidad y alfabetización digital.

1 New Mexico State University. Correo electrónico: binod@nmsu.edu 


\section{INTRODUCCIÓN}

Por lo general, la pedagogía², usada como sinónimo del término enseñanza, es entendida de forma unidimensional, como los métodos de instrucción relacionados con cómo enseñar. Pero esa forma de comprender la pedagogía no muestra su significado con sus múltiples dimensiones las cuales incluyen el componente pedagógico y los contextos. Hoy en día, los aspectos pedagógicos se relacionan con cómo los docentes desarrollan, preparan y practican sus competencias profesionales en la enseñanza y en las aulas de clase (Munby, Russell y Martin, 2001; Shulman, 1986). Las competencias profesionales involucran múltiples componentes pedagógicos que incluyen el conocimiento del contenido, el conocimiento pedagógico y, más recientemente, el conocimiento tecnológico, y el conocimiento generado en la interacción de todos estos componentes (Mishra y Koehler, 2006; Shulman, 1987). Además, la pedagogía no es solo multidimensional sino también un fenómeno complejo que evoluciona de acuerdo con los contextos pedagógicos cambiantes que se dan en la sociedad, en la economía, en la educación y ciencia y tecnología, por mencionar solo algunos aspectos (National Education Technology Plan, 2010). Así, la pedagogía se convierte en un fenómeno dinámico emergente -por lo cual, lo denomino pedagogías emergentes- que requiere una continua renovación de las competencias profesionales de los docentes en contextos pedagógicos cambiantes (como la sociedad, la economía y la tecnología). Desarrollar e implementar pedagogías emergentes en la práctica requiere una comprensión amplia de ambos componentes -pedagógico y contextual-, es decir, la interacción de tecnología, pedagogía, contenido y sociedad.

Esta interacción es evidente e inevitable, especialmente con el crecimiento de la sociedad

2 La noción de pedagogía tiene varias dimensiones que incluyen enseñar, aprender, currículo y evaluación. En este artículo usaré enseñar como el punto focal donde anclo las demás dimensiones, si es necesario. en redes (Castells, 1996) y el uso intensivo de las tecnologías de la información y la comunicación en la sociedad y en las escuelas. Las sociedades en red, penetradas por las tecnologías de los medios (por ejemplo la Web 2.0, los medios y la nube) y el procesamiento de información, que están rápidamente convirtiéndose en una sociedad del conocimiento, y también incrementando su interés por la economía del conocimiento (Andreson, 2008; Castells, 1996; Van Dijk, 2006). La sociedad en redes está comprimiendo (Carnoy y Castells, 2001) la diversidad global en un solo espacio de maneras inesperadas. Por ejemplo, las personas que vienen de todas partes del mundo con diversos trasfondos socioeconómicos, culturales y políticos se ven involucradas en actividades económicas mediadas digitalmente, colaboraciones e interacciones que trascienden límites de tiempo y espacio. Esta compresión de diversidad global también clama por una cuestión de equidad, justicia y libertad relacionada con la existencia y participación de todos los individuos y comunidades, así las prácticas existentes estén orientadas a la diversidad democrática en una sociedad global conectada en redes. Adicionalmente, la sociedad del conocimiento basada en redes, la cual llamaré ahora sociedad del conocimiento en red (NKS, por su sigla en inglés), y la economía del conocimiento también requieren de nuevos tipos de alfabetización -alfabetización digital- para poder participar e interactuar en las actividades socioeconómicas.

Pero en el pasado, los marcos pedagógicos más importantes -por ejemplo, el conocimiento sobre contenido tecnológico-pedagógico (Mishra y Koehler, 2006) y la educación multicultural (Banks, 1993; Bennett, 2001; Gay, 2000)- no se enfocan ni discuten la interrelación entre la tecnología, la pedagogía, el contenido y la sociedad, de una manera u otra. En este contexto, argumento que la pedagogía debería estar enmarcada en las pedagogías emergentes con el fin de incluir su naturaleza emergente y multidimensional, los contextos pedagógicos cambiantes (la sociedad del conocimiento 
en redes, la economía del conocimiento, la democracia orientada a la diversidad y la alfabetización digital), y la interrelación de estos componentes y contextos. Las pedagogías emergentes interrelacionan tecnología, pedagogía, contenidos y sociedad como componentes integrales de las pedagogías. En este artículo, expongo que los marcos pedagógicos existentes, incluyendo el que promueve un conocimiento tecnológico y pedagógico del contenido (TPCK, por su sigla en inglés) y la educación multicultural (MCE, por su sigla en inglés) son problemáticos porque no contemplan los actuales contextos pedagógicos cambiantes. Considero necesario repensar los marcos pedagógicos existentes dentro de los contextos pedagógicos cambiantes. Por lo tanto, me dispongo a explorar y examinar esos contextos pedagógicos cambiantes a los que me refiero incluyendo la sociedad del conocimiento en redes, la economía del conocimiento, la democracia orientada a la diversidad y la alfabetización digital. Exploro estos contextos pedagógicos cambiantes con el fin de derivar implicaciones para las pedagogías emergentes. Por último, teniendo en cuenta las implicaciones, discuto las pedagogías emergentes como concepto y como práctica dirigiendo mi discusión desde las pedagogías repensadas a las pedagogías emergentes.

\section{PROBLEMAS DE LOS MARCOS PEDAGÓ- GICOS EXISTENTES}

Actualmente, y para referirme a la penetrante presencia e integración de la tecnología en las escuelas y salones de clase y a la creciente diversidad estudiantil, existen dos marcos pedagógicos principales que se han implementado ampliamente en los programas de formación de docentes, y en cierta medida en los de su desarrollo profesional. Ellos son: el marco sobre conocimiento tecnológico pedagógico del contenido (Mishra y Koehler, 2006) y la educación multicultural (Banks, 1993; Bennet, 2001; Gay, 2000). Ambos marcos pedagógicos cumplen un papel esencial en el desarrollo, preparación y uso de competencias profesionales y pedagógicas de docentes en servicio.

El marco sobre TPCK fue construido a partir del concepto de conocimiento pedagógico del contenido (PCK, por su sigla en inglés) integrando el componente de tecnología. TPCK establece "las conexiones, interacciones, beneficios y limitaciones entre el contenido, la pedagogía y la tecnología" (Mishra y Koehler, 2006, p. 1025). Además, TPCK provee una base conceptual y teórica esencial para desarrollar y diseñar "estrategias pedagógicas y un lente analítico para estudiar los cambios en el saber de los docentes sobre experiencias de enseñanza exitosas utilizando las tecnologías" (p. 1046). Dada la penetración de la tecnología en las escuelas y en la vida diaria y en la era de enseñar nuevas generaciones de aprendices -denominados nativos digitales (Prensky, 2001), generación de los jóvenes en red (Tapscott, 2008) y niños en internet (Prensky, 2010)-, el marco pedagógico TPCK tiene eco en la idea que los docentes no pueden ya separar o rechazar la tecnología de sus prácticas pedagógicas. Sin embargo, a pesar de su significado y prominencia en la enseñanza y la pedagogía, TPCK es problemático por no incluir el factor pedagógico contextual como importante (por ejemplo, la actual sociedad del conocimiento cambiante) $y$, en cambio, enfocarse en una pedagogía que amplia y desafortunadamente se encuentra desligada de la sociedad, la cultura y la economía.

Desde principios del siglo XXI y hasta nuestros días, la última función y meta de la pedagogía es abastecer la educación para desglozar las necesidades, demandas y cambios de la sociedad contemporánea (Dewey, 1959; Spencer, 2001). Como es comprensible, no podemos estar adicionando componentes uno después de otro (por ejemplo, sociedad, cultura y economía) al modelo de Shulman PCK, pero al mismo tiempo como lo hace TPCK, no podemos ignorar las estructuras que subyacen la pedagogía -lo social, cultural y las estructuras económicas que se relacionan con la educación, la escuela y la pedagogía (Dewey, 1959; Freire, 2000; NETP, 2010)-. La pedagogía, sobre todo con 
la integración de la tecnología, está enfocada en cómo los docentes diseñan los ambientes de aprendizaje que incluyen de manera integrada la comunidad y cómo ellos manifiestan sus competencias profesionales en estos ambientes que involucran a los estudiantes en el aprendizaje (Bransford, Brown y Cocking, 2000; NETP, 2010). Bransford, Brown, y Cocking (2000) consideran que los ambientes de aprendizaje deberían estar centrados en el aprendiz, en el conocimiento, en la evaluación y en la comunidad. Pero TPCK apenas si menciona las estructuras que subyacen la pedagogía (por ejemplo, la estructura social, cultural y económica) a través de las discusiones sobre tecnología, pedagogía y contenido.

Luego, la educación multicultural (MCE) comenzó con el movimiento de derechos civiles de los años 1960 convergiendo intereses políticos del Movimiento de Poder Negro y el Movimiento de Educación Intergrupal (también conocida como educación intercultural) de los años cuarenta y cincuenta (Banks, 1993; Gibson, 1976). Desde entonces, MCE ha expandido su alcance hacia la creación de educación democrática e incluyente para todos, al examinar los contenidos del currículo, los procesos de construcción de conocimiento, los perjuicios predominantes, equidad en la educación, y las dinámicas de poder institucional (por ejemplo, en las escuelas) y las estructuras sociales (Banks, 1993). Hoy, MCE investiga varios aspectos socioculturales de la pedagogía y defiende la equidad educativa y la justicia social para todos los estudiantes, quienes vienen de contextos socioeconómicos y culturales diversos incluyendo raza y etnicidad, género, clase, lenguaje, religión, edad, habilidad y orientación sexual (Adams et al., 2010; Nieto y Bode, 2012, Sleeter y Grant, 1987). Dado el incremento en la diversidad de Estados Unidos, MCE ha ganado prominencia en los programas de educación de docentes y las pedagogías que ayudan a los docentes a desarrollar competencia multicultural (Bennet, 2001) y pedagogías sensibles a la cultura (Gay, 2000).
MCE es esencial para desarrollar, preparar y practicar las competencias profesionales de los docentes y las pedagogías para una sociedad diversa. Sin duda, MCE establece de manera exitosa las conexiones conceptuales y teóricas entre pedagogía y diversidad sociocultural, al reconocer la importancia del conocimiento cultural de los estudiantes, los fondos de conocimiento (Moll, Amanti, Neff y Gonzalez, 1992), el aprendizaje en el aula de clase, mientras se manifiesta en contra de un conocimiento eurocéntrico, monolítico y monolingüe. MCE también promueve enseñanza y aprendizaje culturalmente sensible (Gay, 2000) a través de un aprendizaje constructivista (Brooks y Brooks, 1993; Bruner, 1986; Vygotsky, 1978) y pedagogías críticas (Freire, 2000; Giroux, 1985; Kincheloe y McLaren, 2007), en los cuales los estudiantes construyen significado de las realidades y critican las realidades situándose en sus contextos sociohistóricos respectivos. Pero MCE también tiene problemas mediando los fondos de conocimiento y construyendo significados sociohistóricos de las realidades de los estudiantes dado que MCE tiende a lidiar exclusivamente con problemas de educación sociocultural, económico y político existentes. MCE requiere extender su alcance y explorar y desafiar los contextos emergentes actuales de la sociedad del conocimiento en redes, la economía del conocimiento, la democracia orientada a la diversidad y la alfabetización digital con el fin de promover los fondos de conocimiento de los estudiantes y sus procesos de construcción de conocimiento.

En conclusión, a pesar de que TPCK y MCE proveen a los docentes fundamentos pedagógicos que incluyen contenidos, pedagogía, tecnología y sociedad, estos marcos normalmente se quedan cortos en ver la interrelación entre los componentes pedagógicos y los contextos. TPCK y MCE presentan una dificultad básica: ambos se enfocan en arreglar los problemas socioculturales y profesionales existentes, pero muestran muy poco interés sobre los contextos pedagógicos cambiantes actuales como la sociedad del conocimiento en redes y la economía del conocimiento. 
Aún más, el arreglar permanece en gran parte sin hacer y así mismo permanece la noción de pedagogía poco relevante a un gran número de estudiantes y de contextos pedagógicos cambiantes, si la pedagogía o la enseñanza es para "mediar el aprendizaje de los estudiantes" (Laurillard, 1993) en la sociedad contemporánea. En el presente, en Estados Unidos, doce millones de trabajadores están sin trabajo con un $23 \%$ de jóvenes en edad para trabajar desempleados y los otros millones están subempleados (Bureau of Labor Statistics, 2013); la brecha entre ricos y pobres es la más amplia en los registros de la historia de la humanidad; la salud de las personas y las condiciones de vida están deteriorando, a pesar de las investigaciones e innovaciones en marcha en varios campos; la noción de democracia se ha tornado brumosa con la creciente influencia de dineros corporativos en políticas y gobiernos (Korten, 2001; Ryoo y McLaren, 2010); y el nivel de proficiencia en alfabetización (alfabetización documentada y cuantificada) está deteriorada mientras la alfabetización crítica (por ejemplo, prosa) se mantiene desalentadoramente igual con solo $13 \%$ de proficientes y $14 \%$, aún por debajo del nivel básico (National Center for Educational Statistics, 2003). De manera similar, la alfabetización digital para la economía del conocimiento de hoy y la democracia orientada a la diversidad, enredada en los problemas inherentes sobre la división digital (eSchool News, 2011; Gorski, 2009), están muy lejos del ritmo de los avances de la tecnología. A pesar de la proliferación de tecnología en las escuelas y en la vida diaria, la alfabetización digital apenas recibe algo de atención. Básicamente, la educación y las pedagogías tradicionales están quedando irrelevantes para los aprendices por ofrecer muy poca preparación para conseguir un empleo, alfabetización para el siglo XXI (por ejemplo, alfabetización digital), bienestar individual y un nuevo tipo de democracia emergente.

\section{REFLEXIONANDO EN TPCK, MCE Y LAS PEDAGOGÍAS TRADICIONALES}

Teniendo en cuenta las fallas históricas de la educación descritas, es una realidad que muchas de las pedagogías diseñadas e implementadas tradicionalmente están resultando obsoletas, ya que solo pueden contribuir de forma parcial al cambio social, a garantizar empleo o, incluso, a encontrar el propósito básico de la enseñanza/educación o la construcción de alfabetizaciones. Aunque TPCK y MCE son fundacionales en la pedagogía, son marcos de referencia que por sí mismos no pueden encontrar las necesidades y demandas de la rápida sociedad emergente, que esta ágilmente transformándose en una sociedad de conocimiento en la red que circunscribe nuevos tipos de economía, diversidad y democracia. Desde que esta transformación social empieza a crear nuevas oportunidades y retos pedagógicos (Delanty, 2001; Hargreaves, 2003), es importante explorar la noción de sociedad de conocimiento en la red y los componentes que la constituyen (por ejemplo, economía del conocimiento, diversidad orientada a la democracia y alfabetizaciones digitales) para reflexionar en las pedagogías existentes.

Reflexionar significa explorar nuevos significados de las pedagogías dentro de los contextos pedagógicos contemporáneos (sociedad, cultura, economía y democracia) y los componentes de tecnología, pedagogía y contenido. Reflexionar profundiza en los nuevos significados de las pedagogías y provee un fundamento para que los profesores desarrollen pedagogías transformativas que permitan el avance de la sociedad, la economía, la diversidad, la democracia y las alfabetizaciones; respondiendo a sus necesidades y aspiraciones. Sin embargo, reflexionar también llama a que los profesores entiendan más acerca de los contextos pedagógicos en evolución y tengan una práctica sostenida de exploración de nuevos significados dentro de los contextos. Actualmente, los contextos pedagógicos en evolución incluyen la sociedad 
del conocimiento en red, la economía del conocimiento, la democracia orientada a la diversidad, y las alfabetizaciones digitales. Estos contextos se dan gracias a la relación existente de los componentes pedagógicos (tecnología, pedagogía y contenido) y a los contextos (la sociedad en red), sobre todo con la gran influencia del conocimiento de la sociedad en red y sus elementos constitutivos (la economía del conocimiento).

En síntesis, la sociedad del conocimiento en red (NKS) utiliza la economía del conocimiento para su fundamento (Anderson, 2008; Powell y Snellman, 2008). Y el NKS, en colaboración con la economía del conocimiento, conduce a la diversidad global dentro de un espacio y tiempo interactivo para vincular a todas las personas alrededor del mundo en una amplia variedad de actividades socioeconómicas, comunicaciones y colaboraciones. A su vez, la aproximación global de las personas con sus actividades produce múltiples problemáticas y cuestiones socioculturales, en especial si la existencia y participación de diversos individuos y comunidades son consideradas como justas, equitativas y con prácticas democráticas. En otras palabras, es imperativo explorar a escala mundial, las cuestiones sociales, culturales y económicas de la sociedad en red, siempre y cuando la ética de las personas sea democrática y orientada a la diversidad, al igual que sus prácticas. Por último, NKS y la economía del conocimiento también requieren un nuevo tipo de alfabetización: la digital (Bawden, 2008), para participar e interactuar en entornos socioeconómicos de red, y también establecer prácticas democráticas orientadas a la diversidad en una sociedad global conectada digitalmente. Estos contextos pedagógicos evolutivos no pueden ser separados de las pedagogías pues he mencionado previamente que la principal meta de las pedagogías es satisfacer las necesidades de la educación teniendo en cuenta las demandas y cambios de la sociedad contemporánea (Dewey, 1959; Spencer, 2001). Por tanto, las pedagogías deben acoger a la sociedad-aquí el conocimiento de la sociedad en red es su elemento constitutivo (la economía del conocimiento, la democracia orientada a la diversidad y las alfabetizaciones digitales)-, un componente integral que debe ser considerado como suyo. Abajo describo en detalle cuatro contextos pedagógicos de las pedagogías emergentes: la sociedad del conocimiento en red, la economía del conocimiento, la democracia orientada a la diversidad y las alfabetizaciones digitales enmarcándolas como componentes pedagógicos integrales de las pedagogías emergentes. Presento el NKS como el principal de dichos componentes, ya que los otros tres funcionan como los instrumentos en el entorno del NKS.

\section{COMPONENTES DE LAS PEDAGOGÍAS EMERGENTES}

El primer componente, la noción de sociedad del conocimiento en red, combina las existentes $\mathrm{so}^{-}$ ciedad en red (Castells, 1996; van Dijk, 2006) y la sociedad de conocimiento (Bell, 1973; Unesco, 2005). La combinación de estas dos sociedades construye un nuevo espacio social teniendo estructuras e interacciones nuevas y más eficientes que en la sociedad vista tradicionalmente. La función primaria del nuevo espacio social dentro del NKS es facilitar el procesamiento e intercambio de información para "crear, compartir y discursivisar el conocimiento" (Gurung y Chávez, 2011). En el NKS, crear, compartir y discursivisar el conocimiento están articulados a "las redes sociales y a los medios de comunicación" (van Dijk, 2006). A diferencia de la sociedad tradicional y sus procesos de construcción de conocimiento (principalmente científicas y autorizadas), una sociedad del conocimiento promueve los dos: el conocimiento como ciencia y el conocimiento como cultura (Delanty, 2001). El primero se fundamenta en la racionalidad científica de que el conocimiento viene de la lógica formal, el razonamiento, el experimento y únicamente desde fuentes autorizadas. Por tanto, el conocimiento científico es paradigmático (Kuhn, 1962), universal, rígido, y certero (Ravetz, 1971). Por el contrario, el conocimiento como 
cultura penetra profundamente en "la estructura epistémica de la sociedad" (Delanty, 2001, p. 1) y ratifica la importancia de todos los tipos de conocimiento que vienen de prácticas cotidianas y tradiciones culturales. Desde esta perspectiva, el conocimiento es un recurso "flexible, fluido, en expansión permanente, y constante cambio" (Hargreaves, 2003, p. 16) para ser creado, compartido, y utilizado por personas comunes y corrientes y profesionales. El conocimiento como cultura puede ser descrito como local, específico, y la entidad más práctica que apoya la forma como viven, trabajan, piensan y reflexionan las personas en el diario vivir.

El segundo componente, la economía del conocimiento, apareció a comienzos de la era posindustrial, utilizando, en principio, conocimiento teórico en varias industrias de servicio, tales como servicios financieros y servicios relacionados con la tecnología de la información (Bell, 1973). En el momento, la economía del conocimiento es amplia y se expande rápidamente en su campo, sobre todo con la llegada de las tecnologías de la información, la comunicación, la economía global y la sociedad en red. La economía del conocimiento fundamenta en la ciencia, la tecnología y el capital intelectual (Anderson, 2008; Powell y Snellman, 2004) con la idea de que "el conocimiento como recurso es una comodidad compartible y portable" para mantener y crear nuevas estructuras económicas, libres de "restricciones de tiempo y espacio" (Mioduser, Nachmias y Forkosh-Baruch, 2008, pp. 24-25). Powell and Snellman (2004) establecen que la economía del conocimiento, como conjunto de productos y servicios que se basan en las actividades de conocimiento intensivo, contribuyen a un paso acelerado de avances tecnológicos y científicos, pero también afirman que a su vez estos rápidamente se tornan obsoletos. Los principales componentes de la economía del conocimiento incluyen un mayor grado de dominio en las capacidades intelectuales que en las contribuciones físicas o los recursos naturales, combinados con los esfuerzos de integrar mejoras en cada etapa de los procesos de producción, desde el laboratorio de investigación y desarrollo (I+D), la planta de producción, hasta la interface con los clientes (p. 121).

La economía del conocimiento es recíproca con el NKS mientras se aprovechan las mayores infraestructuras adyacentes (redes sociales, tecnológicas y técnicas) como entradas para desarrollar sus actividades económicas y financieras, y utilizar el conocimiento como un producto básico o una materia prima. Simultáneamente, la economía del conocimiento también racionaliza de forma económica y monetaria, al NKS para motivar la creación y difusión del conocimiento.

El tercer componente, la noción de democracia orientada a la diversidad, refleja un mundo que rápidamente se reduce, al igual que la densidad de la diversidad concentrada en las actividades socioeconómicas y las interacciones en la sociedad del conocimiento en la red así como la economía del conocimiento. La diversidad incluye una gama de grupos socioeconómicos, culturales y políticos, basados en raza, etnia, género, clase, lengua, religión, edad, habilidad, y orientación sexual. La diversidad también incluye grupos categóricos como personas que son agrupadas por su profesión y actividades de interés común (deportes, pasatiempos e intereses). Sin embargo, para el NKS, la economía del conocimiento, las pedagogías emergentes, las diversidades socioeconómicas y culturales se destacan sobre la diversidad categórica. La democracia orientada a la diversidad es importante, sobre todo en los contextos actuales del NKS y la economía del conocimiento, donde las personas de todo el mundo, que tengan diversos trasfondos socioeconómicos, culturales, y políticos, participen para crear, compartir y discursivisar conocimiento (Gurung y Chávez, 2011). En la democracia orientada a la diversidad, los individuos y comunidades participantes deben buscar su compromiso cívico expresando "una variedad de opiniones políticas en cualquier medio" (Bollen y Paxton, 2000, p. 60), hablando con libertad de su identidad individual y grupal, 
sus grupos políticos, y causas comunes o intereses colectivos (Clemens, 1997). De esta manera, los individuos y comunidades participantes deberán simultáneamente la presencia, participación e interacción de sí mismos y de los demás por las posibles implicaciones de ideologías hegemónicas y prácticas opresivas; buscando equidad y justicia en todos los tipos de actividades e interacciones dentro del NKS y la economía del conocimiento.

Por último, la noción de alfabetizaciones digitales (Gilster, 1997; Lankshear y Knobel, 2008), con frecuencia usadas indistintamente con las alfabetizaciones del siglo XXI (cf. New Media Consortium, 2005), incluyen el conocimiento necesario del individuo, sus competencias y actitudes hacia el uso de la tecnología para conocer sus necesidades y propósitos dentro de la actual sociedad del conocimiento y la economía del conocimiento en línea. El término alfabetización digital, presentado por Gilster (1997), como "la habilidad de entender y usar la información en múltiples formatos desde un amplio rango de recursos cuando esta se presenta a través de las computadoras" (p. 1), se ha expandido desde entonces. Hoy en día, las alfabetizaciones digitales se entienden como un complejo conjunto de habilidades tecnológicas que incluyen diferentes tipos de conocimiento técnico y sobre hardware y software (Bawden, 2008). De acuerdo con Bawden (2008), las alfabetizaciones digitales tienen cuatro componentes relacionados que se resumen a continuación:

- Fundamentos: los conocimientos básicos en computación y las alfabetizaciones de las TIC requeridas como herramientas y habilidades básicas en los lugares de trabajo y la sociedad.

- Conocimientos previos: conocimiento requerido acerca de los tipos y naturaleza digital de la información y sus recursos.

- Competencias centrales: habilidades básicas y competencias para leer y entender información en formatos digitales y no digitales, ensamblaje de conocimiento, evaluar información, crear y comunicar información digital, alfabetización sobre el uso de medios, etc.

- Actitudes y perspectivas: la habilidad de desarrollar un aprendizaje independiente y mantener una alfabetización social y moral cuando se vincula al mundo digital.

Asimismo, las alfabetizaciones digitales son emergentes en naturaleza requiriendo nuevos conjuntos de habilidades funcionales y conocimientos críticos para la lectura, escritura y la interacción con los medios digitales, la red y las comunidades en línea mediadas por la red. La tecnología en constante avance implica un aprendizaje continuo y adaptativo de nuevas habilidades tecnológicas y un pensamiento crítico. Especialmente, con la compresión de la diversidad en la sociedad global en línea y la economía del conocimiento, tener una perspectiva crítica es importante dentro de las alfabetizaciones digitales para desmitificar las ideologías nocivas escondidas en el contenido digital (textos, imágenes, videos, etc.) e interacciones. Las perspectivas críticas pueden generarse incrustando teorías tecnológicas y sociales críticas (Feenberg, 1991) tales como alfabetizaciones críticas de los medios de comunicación (cf. Kellner y Share, 2007) y alfabetizaciones críticas dentro de alfabetizaciones digitales (Watulak y Kinzer, 2012). Aunque este es un marco emergente, Watulak y Kinzer (2012) establecen que las alfabetizaciones críticas digitales tienen cuatro elementos centrales: "El entendimiento de los contextos culturales, sociales e históricos del uso de la tecnología, incluyendo practicas éticas y apropiadas; pensamiento crítico y análisis, practica reflexiva, y habilidades funcionales con las tecnologías digitales" (p. 135). Por tanto, las alfabetizaciones críticas digitales comprenden el conocimiento tecnológico, epistémico y crítico esencial, al igual que las habilidades para el uso determinado de las tecnologías contemporáneas en crear, compartir y discursar el conocimiento que es pertinente al NKS, la economía del conocimiento y las pedagogías emergentes. 


\section{RECONSIDERANDOPEDAGOGÍASHACIA EL AUGE DE NUEVAS PEDAGOGÍAS}

Las descripciones realizadas previamente sobre los contextos evolutivos de la sociedad del conocimiento en línea, la economía del conocimiento, la democracia orientada a la diversidad y las alfabetizaciones (críticas) digitales apuntan a creer que reconsiderar una pedagogía involucra prácticas dinámicas, emergentes, situadas y reflexivas. Dinámicas y emergentes, debido a que reflexionar invita a los docentes a que tengan la habilidad de cambiar y adaptar sus pedagogías con los contextos evolutivos y, en consecuencia, tener la intención pedagógica de poner estos cambios en práctica. Situada y reflexiva, porque la meta principal de reflexionar es mejorar, por no decir transformar, las pedagogías locales de los docentes (por ejemplo, las propias) y sus prácticas en el salón de clase, pensando acerca de lo que se hace en los ámbitos local y global, y lo que se puede hacer para transformarlo dentro de los contextos cambiantes de las pedagogías (por ejemplo, el NKS y la economía del conocimiento).

Reflexionar pretende capturar la esencia o los significados de los contextos de las pedagogías cambiantes y ayuda a que los docentes desarrollen prácticas transformadoras de enseñanza que contribuyan al avance de la sociedad, la economía y la democracia, respondiendo a las necesidades actuales. Sin embargo, para responder y avanzar a la cambiante sociedad actual, a su economía y democracia, las pedagogías se convierten en prácticas dinámicas que requieren nuevas reflexiones de forma periódica. Por esta razón, la noción de pedagogías se debe enmarcar, primero como pedagogías emergentes que involucran prácticas re-evaluadas y transformadoras, y rutinizar nuevas reflexiones que conlleven a cambios conceptuales y prácticos en las pedagogías existentes. Posteriormente, describo la noción de pedagogías emergentes en los órdenes conceptual y práctico. En el conceptual, describo las características subyacentes o estructuras de pedagogías emergentes, y en el práctico, discuto las prácticas de enseñanza dentro de las pedagogías emergentes.

\section{PEDAGOGÍAS EMERGENTES COMO CONCEPTO}

Una pedagogía emergente comienza con un enfoque re-evaluador que apunta a explorar nuevos significados de las pedagogías tradicionales existentes dentro de los contextos evolutivos de la sociedad del conocimiento en línea, de la economía del conocimiento, la diversidad orientada a la democracia y las alfabetizaciones (críticas) digitales. Una pedagogía emergente instiga a cambios documentados y visionarios, ubica los problemas de las pedagogías tradicionales y da paso a nuevas pedagogías Se considera también que una pedagogía emergente incorpora pluralidad, fluidez y surgimiento dentro de sí misma.

Como concepto, una pedagogía emergente incorpora las seis características básicas ${ }^{3}$ inmersas en las esencias o significados de los actuales contextos pedagógicos evolutivos (y ahora nuevos componentes pedagógicos) descritos anteriormente, en los cuales se menciona que:

- Integra un alto nivel del uso de la tecnología con las pedagogías.

- Es una praxis pedagógica transformativa.

- Practica interseccionalidad.

- Promueve el aprendizaje continuo y colaborativo.

- Adopta y fomenta el cambio.

Primero, una pedagogía emergente involucra re-pensar pedagogías dentro de los contextos pedagógicos evolutivos: explora y examina las pedagogías tradicionales buscando su significado en los contextos de las sociedades de conocimiento

3 Como la noción de pedagogía emergente es emergente y situada, las características que he proporcionado aquí funcionan como sus fundamentos, no como las limitaciones. La fluidez debe ser imaginada por pedagogía emergente sobre la base de los resultados de la reconsideración de uno mismo. 
en línea, economía del conocimiento, diversidad orientada a la democracia y las alfabetizaciones críticas digitales. Una pedagogía emergente re-imagina a los profesores y estudiantes dentro del NKS no solo como consumidores del conocimiento y trabajadores del conocimiento, como muchas teorías populares afirman (cf. Castells, 1996; Delanty, 2001), sino como creadores de conocimiento. Para docentes y estudiantes, el NKS facilita espacios digitales y herramientas para crear, compartir y discursar el conocimiento. Similarmente, la pedagogía emergente busca empoderar a los estudiantes para la economía del conocimiento, preparándolos como trabajadores altamente calificados, consumidores, críticos, y creadores de conocimiento. El empoderamiento de los estudiantes involucra construir competencias -conocimiento y herramientas- del siglo XXI ${ }^{4}$ (cf. New Media Consortium, 2005) apuntando a cumplir con la, por así decirlo, brecha del ingenio (Homer-Dixon, 2000). La ejecución de esta brecha del ingenio solicita a los docentes y estudiantes de esta generación entender la complejidad de las crisis socioeconómicas y ambientales, y resolverlas responsable y creativamente. Posteriormente, la pedagogía emergente busca promover la democracia orientada a la diversidad, examinando la naturaleza, producción y divulgación del conocimiento, así como las estructuras en la sociedad del conocimiento en línea y en la economía del conocimiento. A este respecto, las pedagogías emergentes deberían investigar los trabajos conectados globalmente, sitios colaborativos y otros espacios interactivos por posibles imbricaciones de dinámicas de poder jerárquico que socaven o amenacen la presencia, participación e interacción de diversas personas y comunidades que vengan de diversos contextos socioculturales como cultura, raza, etnicidad, género, clase, lengua, religión, edad, habilidad y orientación sexual. También, las pedagogías emergentes también construyen, fomentan, y forjan alfabetizaciones críticas

4 Ver Marco de Aprendizaje del Siglo XXI en: http://www.p21.org/ overview/skills-framework digitales para docentes y estudiantes, buscando involucrarlos de manera crítica y significativa en el NKS, la economía del conocimiento y la diversidad orientada a la democracia. En concurrencia con las alfabetizaciones críticas digitales, una pedagogía emergente debería ayudar a docentes y estudiantes a desarrollar conocimiento y habilidades para generar crítica hacia la estructura y organismos de la tecnología (Archer, 2003) sobre cómo las estructuras tecnológicas y los funcionamientos moldean sus identidades digitales como el alter ego (Turkle, 1984), hábitos digitales, pensamiento, y otras capacidades para actuar en las sociedades del conocimiento en línea mediadas digitalmente, la economía del conocimiento y la democracia orientada a la diversidad.

Segundo, una pedagogía emergente integra un uso de alto nivel de la tecnología con las pedagogías: a diferencia de las tradicionales, una pedagogía emergente no puede ser concebida sin la colaboración de la instrumentalidad, teniendo en cuenta que la tecnología se integra con las PE como una forma de mediación (Wertsch, 1998), ampliando y generando nuevas realidades como oportunidades nuevas de aprendizaje. Una PE explora nuevas estructuras y organismos de la tecnología (por ejemplo, Web 2.0, los medios de comunicación y la computación en la nube) para la enseñanza y el aprendizaje. Sin embargo, la integración de la tecnología dentro de las pedagogías emergentes no debería verse afectada por el bajo nivel de uso de la tecnología, como el ejercicio y la práctica, la búsqueda de información y el uso de los computadores como medio de consulta enciclopédica (Cuban, Kirkpatrick y Peck, 2001; Wenglinsky, 1998). En lugar de esto, una pedagogía emergente debería incentivar el alto uso de la tecnología (Cuban, Kirkpatrick y Peck, 2001) utilizándola como instrumento mental o sociointelectual (Jonassen, 2006) para la creatividad, la colaboración y la productividad multimedia. Sin embargo, una pedagogía emergente no asume que la tecnología pueda resolver por completo todo, un determinismo tecnológico, como es retratado 
por la mayoría de los discursos sobre los nativos digitales y generaciones de red (Friedman, 2005; Negroponte, 1995; Prensky, 2001; Tapscott, 2008).

Tercero, una pedagogía emergente es una praxis pedagógica transformativa: inherente a la PE está la reflexión o la práctica reflexiva. La práctica es reflexionar dentro de nuestras propias palabras y acciones, y encontrar nuevos conjuntos de prácticas informadas por la reflexión (Freire, 2000) que transformen problemas pedagógicos en posibilidades (cf. Hooks, 1994). Hay muchos marcos de referencia de teorías críticas que buscan prácticas pedagógicas transformativas como: las pedagogías críticas (Freire, 2000; Giroux, 1985; McLaren, 1995), la educación crítica multicultural (May y Sleeter, 2010), teorías críticas de raza ${ }^{5}$ en la educación (Delgado y Stefancic, 2001; Kendall, 2006; Ladson-Billings y Tate, 1995; Taylor, Gillborn y Ladson-Billings, 2009), pedagogías feministas (Finke, 1994; Lather, 1991), aprendizaje transformativo (Mezirow, 1991), reproducción cultural (Bourdieu y Passeron, 2000) o las teorías del pos-(posmodernismo, poscolonialismo, posestructuralismo, etc.). Una pedagogía emergente utiliza estos marcos de referencia, de manera individual o multidisciplinarmente, para interpretar significados de las pedagogías en contextos, generando crítica y problematizando las pedagogías dentro de los contextos NKS, economía del conocimiento, democracia orientada a la diversidad y las alfabetizaciones críticas digitales que son descritas arriba.

Cuarto, una pedagogía emergente practica la interseccionalidad; es decir, busca proactivamente posibles intersecciones pedagógicas dentro de sus contextos y componentes pedagógicos (sociedad del conocimiento en línea, economía del conocimiento, democracia orientada a la diversidad y las alfabetizaciones críticas digitales). Las intersecciones pueden incluir un conjunto de actividades reflexivas, como la búsqueda de intersecciones, conexiones y convergencias entre los contextos y

5 La teoría crítica sobre raza, va más allá de la dualidad entre blanco y negro, se extiende a los asiáticos, latinos e indígenas. componentes pedagógicos y en consecuencia los contenidos prácticos de enseñanza que se facilitan.

Quinto, una pedagogía emergente incentiva al aprendizaje continuo y colaborativo. No puede ser desarrollada y practicada sin tener un concepto de aprendizaje continuo. Debido a que todos los componentes de una pedagogía emergente, incluyendo tecnología, pedagogía, contenido y sociedad están en evolución, los docentes necesitan desarrollar una experiencia adaptativa -cambiando la experiencia pero fundamentada en reflexionar e investigar- hasta comprender cómo estos componentes interactúan e influyen en sus propias pedagogías. Es importante notar que el desarrollo de la experiencia adaptativa es un concepto relativo, la experiencia de una persona se revela solo cuando se comparte y se discursa a través de la colaboración dentro de comunidades profesionales (por ejemplo, equipos de desarrollo profesional, conferencias y seminarios).

Por último, una pedagogía emergente adopta e incentiva al cambio; involucra prácticas tanto informadas como visionarias. En la actualidad, las pedagogías se convierten cada vez más en prácticas que solo son informadas (por ejemplo, evidencia/ enseñanza basada en la investigación), mientras se desplazan gradualmente los aspectos visionarios de la educación. (cf. Dewey, 1959). El simple enfoque en las pedagogías como prácticas informadas es problemático en el sentido que estas solo responden a problemas o crisis existentes, mientras se cierran puertas a posibilidades inimaginables de y para el futuro, que pretenden el cambio que tanto se necesita.

\section{PEDAGOGÍAS EMERGENTES COMO PRÁCTI- CA: ENSEÑANZA CON PEDAGOGÍAS EMERGENTES}

Enseñar con pedagogías emergentes comienza con propósitos renovados y metas inmersas en sus propias características, e informadas por el conocimiento epistémico y tecnológico, y las habilidades generadas mediante la reflexión y la investigación. 
Enseñar con pedagogías emergentes es complejo, pero también es una práctica pedagógica emocionante. La complejidad se da por la actual sociedad del conocimiento en red en la cual los maestros deben capturar sus intereses e imperativos competitivos (Hargreaves, 2003, p. 10), especialmente las nuevas tecnologías y las expectativas de la sociedad de crear, compartir y discursar el conocimiento. En primer lugar, la sociedad conectada (Castells, 1996) demanda un alto nivel de uso de la tecnología en las aulas de clase (Cuban, Kirkpatrick y Peck, 2001). Integrar la tecnología, como se ha hecho hasta ahora, se convirtió en un proceso desordenado (Zhao, Pugh, Sheldon y Byers, 2002) y puede ser un reto para los docentes que desarrollan nuevos sets de conocimiento, habilidades y actitudes para superar sus propias barreras internas (es el caso de las creencias y actitudes sobre la tecnología) y externas (la brecha digital y las políticas escolares). En segundo plano, la práctica de las pedagogías emergentes como parte de NKS puede ser un reto, teniendo en cuenta que esto implica reflexionar sobre y desarrollar pedagogías que promuevan la creatividad y la imaginación de los estudiantes para llenar la brecha del ingenio (Homer-Dixon, 2000). De igual manera, la práctica de las pedagógicas emergentes engloba una comprensión clara de otros contextos cambiantes incluyendo la economía del conocimiento, la democracia orientada a la diversidad y las alfabetizaciones digitales críticas.

De otra parte, enseñar utilizando las pedagogías emergentes es una práctica pedagógica emocionante, si se tienen en cuenta los beneficios educativos de la tecnología y las nuevas generaciones de estudiantes quienes están creciendo digitales como la generación de los jóvenes en red (Tpascott, 2008) y nativos digitales o niños en red (Prensky, 2001, 2010). Los docentes pueden lograr un alto nivel de participación en el aprendizaje, y posiblemente de entusiasmo, en el aula de clase, si aprovechan los beneficios multimodales de la tecnología, como los hipertextos, imágenes, videos, juegos en línea y videojuegos, materiales multimedia y computación en la nube. Con el alto uso de la tecnología, los docentes pueden transformar la instrucción tradicional unidireccional (del docente hacia los estudiantes) en ricas experiencias de aprendizaje multidireccionales a través de la creatividad y la colaboración, como la creación colaborativa, compartir y discursar el contenido de aprendizaje en formas multimodales. Además, con las nuevas tecnologías como la Web 2.0 (Greenhow, Robelia y Hughes, 2009; Gurung y Chávez, 2011) los medios sociales (Dabbagh y Kitsantas, 2012), la computación en la nube (Limbu, 2012; Warschauer, 2011), y otras propiedades de las tecnologías educativas, la enseñanza y el aprendizaje pueden trascender la enseñanza tradicional del aula, convirtiendo el aprendizaje en algo que puede suceder en cualquier momento, en cualquier lugar, a través de cualquier dispositivo, bajo demanda y que puede ser aprendido por todos. De igual manera, los estudiantes de hoy son nativos digitales o niños en internet que no solamente están rodeados por tecnología digital (computadores, tabletas, teléfonos celulares, MP3, videojuegos y otros juguetes o herramientas de la edad digital) (Prensky, 2001, p. 1), sino que ellos naturalmente interactúan en un ambiente tecnológico y crean conocimiento sofisticado y destrezas al usar las tecnologías, sin dificultad (Nasah, DaCosta, Kinsell y Seok, 2010; Prensky, 2010; Tapscott, 2008). De tal manera que la combinación entre los beneficios de las nuevas tecnologías educativas y el nacimiento de estudiantes con herramientas digitales y ambientes, los docentes pueden diseñar nuevas e interesantes horizontes ${ }^{6}$ que no fueron posibles en el pasado.

\section{CONCLUSIONES E IMPLICACIONES}

Las pedagogías emergentes, al capturar las fortalezas y debilidades de los marcos pedagógicos existentes más nombrados, incluyendo TPCK y MCE, buscan establecer una interrelación entre la

6 Por ejemplo, el aprendizaje basado en juegos, el aprendizaje virtual y el aprendizaje en línea. 
tecnología, la pedagogía, el contenido y la sociedad. Aquí sostengo que la noción de pedagogías no es estática, y por tanto, debería estar enmarcada como pedagogías emergentes, reflejando un panorama dinámico y cambiante de los contextos pedagógicos actuales (por ejemplo, la sociedad del conocimiento en red, la economía del conocimiento, la democracia orientada a la diversidad y las alfabetizaciones digitales). El desarrollo de las pedagogías emergentes comienza con el repensar las pedagogías tradicionales existentes y el buscar la interacción entre los contextos pedagógicos (la sociedad del conocimiento en red, etc.) y los componentes (tecnología, pedagogía, contenidos y sociedad).

Las pedagogías emergentes tienen un potencial promisorio para combinar tecnología, educación, diversidad, democracia, sociedad del conocimiento y economía del conocimiento; de este modo, persiguen un compromiso significativo de los estudiantes que se ajuste a los estudiantes de hoy, como juventud digital, nativos digitales y niños internet (Prensky, 2001, 2010) -quienes tendrán que trabajar colaborativamente e interactuar con una gran diversidad de personas, y quienes tendrán que crear, compartir y discursar el conocimiento en el día a día-. Sin embargo, el trato amplio que le he dado a las pedagogías emergentes en este artículo es solamente una comprensión de sus potenciales pedagógicos (Dede y Richards, 2012). Se necesita más diálogos teóricos, investigación y práctica para hacer progresar el concepto y la práctica de las pedagogías emergentes. Los diálogos teóricos deberían problematizar y poner las pedagogías emergentes en perspectiva. La investigación y la práctica deberían instigar el proceso de explicar e interpretar las pedagogías emergentes para el desarrollo e implementación exitosa en las prácticas de enseñanza en el aula de clase. De esta manera, sugiero que las investigaciones y las teorías futuras deberían establecer tensiones creativas entre las pedagogías emergentes como concepto y como práctica, del concepto y la práctica de las pedagogías emergentes que yo he discutido en este artículo.

\section{REFERENCIAS}

Adams, M.; Blumenfeld, W.J.; Casteneda, R.; Hackman, H.W.; Peters, M.L. y Zuniga, X. (eds.) (2010). Readings for Diversity and Social Justice: An Anthology on Racism, Antisemitism, Sexism, Heterosexism, Ableism, and Classism. 2a ed. Nueva York: Routledge

Anderson, R.E. (2008). Implications of the Information and Knowledge Society for Education. En: J. Voogt y G. Knezek (eds.). International Handbook of Information Technology in Primary and Secondary Education (pp. 5-22). Nueva York: Springer.

Archer, M.S. (2003). Structure, Agency, and the Internal Conversation. Cambridge: Cambridge University Press.

Banister, S. y Reinhart, R. V. (2011). TPCK for impact: Classroom teaching practices that promote social justice and narrow the digital divide in an urban middle school. Computers in the Schools, 28(1), 5-26.

Banks, J.A. (1993). Multicultural Education: Historical Development, Dimensions, and Practice. Review of Research in Education, 19, 3-49.

Bawden, D. (2008). Origins and Concepts of Digital Literacy. En: C. Lankshear y M. Knobel (eds.). Digital Literacies: Concepts, Policies, and Practices (pp. 1732). Nueva York: Peter Lang.

Bell, D. (1973). The Coming of Post-Industrial Society: A Venture in Social Forecasting. Nueva York: Basic Books.

Bennett, C. (2001). Genres of Research in Multicultural Education. Review of Educational Research, 71(2), 171-217.

Bollen, K.A. y Paxton, P. (2000). Subjective Measures of Liberal Democracy. Comparative Political Studies, $33(1), 58-86$

Bourdieu, P. y Passeron, J-C. (2000). Reproduction in Education, Society and Culture. 2a. ed. Thousand Oaks, CA: Sage. 
Bransford, J.D.; Brown, A.L. y Cocking, R.R. (eds.) (2000). How People Learn: Brain, Mind, Experience, and School. Washington D.C.: National Academy Press

Brooks, L. y Brooks, M. (1993). In Search of Understanding: The Case for Constructivist Classrooms. 2a. ed. Alexandria, VA: Associate for Supervision and Curriculum Development (ASCD).

Bruner, J. (1986). Actual Minds, Possible Minds. Cambridge, MA: Harvard University Press.

Bureau of Labor Statistics (2013). The Employment Situation - 2013. Washington, DC: U.S. Department of Labor. Recuperado el 15 de febrero de 2013 de: http://stateofworkingamerica.org /charts/ underemployment-gender/

Carnoy, M. y Castells, M. (2001). Globalization, the Knowledge Society, and the Network State: Poulantzas at the Millennium. Global Networks, 1(1), $1-18$.

Castells, M. (1996). The Rise of the Network Society. Vol. 1. Oxford: Blackwell.

Clemens E. (1997). The People's Lobby: Organizational Innovation and the Rise of Interest Group Politics in the United States, 1890-1925. Chicago, IL: University of Chicago Press

Cuban, L.; Kirkpatrick, H. y Peck, C. (2001). High Access and Low Use of Technologies in High School Classrooms: Explaining an Apparent Paradox. American Educational Research Journal, 38(4), 813-834.

Dabbagh, N. y Kitsantas, A. (2012). Personal Learning Environments, Social Media, and Self-Regulated Learning: A Natural Formula for Connecting Formal and Informal Learning. Internet and Higher Education, 15(1), 3-8.

Dede, C. y Richards, J. (eds.). (2012). Digital Teaching Platforms. Nueva York: Teacher's College Press.

Delanty, G. (2001). Challenging Knowledge: The University in the Knowledge Society. Filadelfia, PA: The Society for Research into Higher Education y Open University Press.

Delgado, R. y Stefancic, J. (2001). Critical Race Theory: An Introduction. Nueva York: New York University Press.
Dewey, J. (1956). The Child and the Curriculum. Chicago, IL: The University of Chicago Press.

Ertmer, P.A. (2005). Teacher pedagogical beliefs: The final frontier in our quest for technology integration? Educational Technology Research and Development, 53(4), 25-39.

eSchool News (enero de 2011). For Minorities, New 'Digital Divide' Seen. TechnologyNewsforToday'sK-20Educator. Recuperadodehttp://www.eschoolnews.com/2011/01/11/ for-minorities-newdigital-divide-seen/

Feenberg, A. (1991). Critical Theory of Technology. Nueva York: Oxford University Press.

Finke, L.A. (1994). A Pedagogy of the Depressed: Feminism, Post-Structuralism, and Pedagogical Practice. En: D.F. Sadoff y E.C. William (eds.). Teaching Contemporary Theory to Undergraduates (pp. 155-168). Nueva York: Modern Language Association of America.

Freidman, T. (2005). The World is Flat. Nueva York: Farrar, Straus y Giroux.

Freire, P. (2000). Pedagogy of the Oppressed. Nueva York: Continuum.

Gay, G. (2000). Culturally Responsive Teaching: Theory, Research, and Practice. Nueva York: Teachers College Press.

Gibson, M. A. (1976). Approaches to Multicultural Education in the United States: Some Concepts and Assumptions. Anthropology and Education Quarterly, 7, 7-18.

Gilster, P. (1997). Digital Literacy. Nueva York: John Wiley y Sons.

Giroux, H. (1985). Critical Pedagogy, Cultural Politics and the Discourse of Experience. Journal of Education, 167(2), 22-41.

Gorski, P.C. (2009). Insisting on Digital Equity Reframing the Dominant Discourse on Multicultural Education and Technology. Urban Education 44(3), 348-364. DOI: 10.1177/0042085908318712.

Greenhow, C.; Robelia, B. y Hughes, J.E. (2009). Learning, Teaching, and Scholarship in a Digital Age: Web 2.0 and Classroom Research: What Path Should We Take Now? Educational Researcher, 38(4), 246-259. 
Gurung, B. y Chávez, R. C. (2011).Transformative Multicultural Engagement on a Web 2.0 Interface: Forging a Multicultural Education 2.0. En: G. Kurubacak y T.V. Yuzer (eds.). Handbook of Research on Transformative Online Education and Liberation: Models for Social Equality (pp. 15-46). Hershey, PA: IGI Global.

Hargreaves, A. (2003). Teaching in the Knowledge Society: Education in the Age of Insecurity. Nueva York: Teachers College Press

Hargreaves, A. (2003). Teaching in the Knowledge Society: Education in the Age of Insecurity. Nueva York: Teacher College Colombia University Press.

Homer-Dixon, T.F. (2000). The Ingenuity Gap. Nueva York: Knopf.

Hooks, B. (1994). Teaching to Transgress: Education as the Practice of Freedom. Nueva York: Routledge

Jonassen, D.H. (2006). Modelling with Technology: Mindtools for Conceptual Change. 3a. ed. Columbus, $\mathrm{OH}$ : Merrill/Prentice Hall.

Kellner, D. y Share, J. (2007). Critical Media Literacy: Crucial Choices for a Twenty-First-Century Democracy. Policy Futures in Education, 5(1), 59-69.

Kendall, F.E. (2006). Understanding White Privilege: Creating Pathways to Authentic Relationships across Race. Nueva York: Routledge.

Kincheloe, J.L. y McLaren, P. (eds.) (2007). Critical Pedagogy in the Twenty-First Century: Evolution for Survival. Nueva York: Peter Lang.

Korten, D.C. (2001). When Corporations Rule the World. 2a. ed. San Francisco, CA: Berrett-Koehler Publishers

Kuhn, T.S. (1962). The Structure of Scientific Revolutions. Chicago, IL: University of Chicago Press.

Ladson-Billings, G. y Tate, W. (1995). Toward a Critical Race Theory of Education. Teachers College Record 97(1), 47-68.

Lankshear, C. y Knobel, M. (eds.) (2008). Digital Literacies: Concepts, Policies, and Practices. Nueva York: Peter Lang.

Lather, P. (1991). Getting Smart-Feminist Research and Pedagogy within the Postmodern. Nueva York: Routledge.
Laurillard, D. (1993). Rethinking University Teaching: A Framework for the Effective Use of Educational Technology. Nueva York: Routledge.

Limbu, M. (2012). Teaching Writing in the Cloud: Networked Writing Communities in the Culturally and Linguistically Diverse Classrooms. Journal of Global Literacies, Technologies, and Emerging Pedagogies, 1(1), 1-20.

May, S. y Sleeter, C. (eds.). (2010). Critical Multiculturalism: Theory and Praxis. Nueva York: Routledge.

McLaren, P. (1995). Critical Pedagogy and Predatory Culture: Oppositional Politics in a Postmodern Era. Nueva York: Routledge.

Mezirow, J. (1991). Transformative Dimensions of Adult Learning. San Francisco: Jossey-Bass.

Mioduser, D.; Nachmias, R. y Forkosh-Baruch, A. (2008). New Literacies for the Knowledge Society. En: J. Voogt y G. Knezek (eds.). International Handbook of Information Technology in Primary and Secondary Education (pp. 23-42). Nueva York: Springer.

Mishra, P. y Koehler, M.J. (2006). Technological Pedagogical Content Knowledge: A Framework for Teacher Knowledge. Teachers College Record, 108(6), 1017-1054

Moll, L.C.; Amanti, C.; Neff, D. y Gonzalez, N. (1992). Funds of Knowledge for Teaching: Using a Qualitative Approach to Connect Homes and Classrooms. Theory into Practice, 31(2), 132-141.

Munby, M.; Russell, T. y Martin, A.K. (2001). Teachers' Knowledge and How It Develops. En: V. Richardson (ed.). Handbook of research on teaching (pp. 877-904). Washington, DC: American Educational Research Association.

Nasah, A.; DaCosta, B.; Kinsell, C. y Seok, A. (2010). The Digital Literacy Debate: An Investigation of Digital Propensity and Information and Communication Technology. Education Technology Research and Redevelopment, 58(5), 531-555

National Center for Education Statistics (2003). National Assessment of Adult Literacy National Center for Education Statistics. (2006). The nation's report card: Reading 2005. Recuperado el 29 de 
noviembre de 2009 de: nces.ed.gov/nationsreportcard/pdf/main2005/2006451.pdf

National Education Technology Plan (2010). Transforming American Education Learning: Powered by Technology. Washington, DC.

Negroponte, N. (1995). Being Digital. Nueva York: Alfred Knopf.

New Media Consortium (2005). A Global Imperative: The Report of the 21st Century Literacy Summit. Austin, TX: New Media Consortium. Recuperado el 11 de febrero de 2013 de: http://www.nmc.org/ pdf/Global_Imperative.pdf

Nieto, S. y Bode, P. (2012). Affirming Diversity: The Sociopolitical Context of Multicultural Education. 6a. ed. Upper Saddle River, NJ: Pearson Education, Inc.

Powell, W.W. y Snellman, K. (2004). The Knowledge Economy. Annual Review of Sociology, 30, 199-220.

Prenksy, M. (2001). Digital Natives, Digital Immigrants. On the Horizon, 9(5), 1-6.

Prensky, M. (2010). Teaching Digital Natives: Partnering for Real Learning. CA: Corwin Press.

Ravetz, J.R. (1971). Scientific Knowledge and its Social Problems. Oxford: Clarendon Press

Ryoo, J.J. y McLaren, P. (2010). Seeking Democracy in American Schools: Countering Epistemic Violence through Revolutionary Critical Pedagogy. En: F. Salili y R. Hoosain (eds.). Democracy and Multicultural Education (pp. 99-132). Charlotte, NC: Information Age Publishing.

Sleeter, C.E. y Grant, C.A. (1987). An Analysis of Multicultural Education in the United States. Harvard Educational Review, 57(4), 421-444.

Spencer, D. (2001). Teachers' Work in Historical and Social Context. En: V. Richardson (ed.). Handbook of Research on Teaching (pp. 803-825). 4a. ed. Washington, DC: American Educational Research Association.
Shulman, L.S. (1986). Those Who Understand: Knowledge Growth in Teaching. Educational Researcher, 15(2), 4-14.

Shulman, L.S. (1987). Knowledge and Teaching: Foundations of the New Reform. Harvard Educational Review, 57(1), 1-22.

Taylor, E.; Gillborn, D. y Ladson-Billings, G. (2009). Foundations of Critical Race Theory in Education. Nueva York: Routledge/Taylor y Francis Group.

Tapscott, D. (2008). Grown Up Digital: How the Net Generation is Changing Your World. Nueva York: McGraw-Hill.

Turkle, S. (1984). The second self. Computers and the Human Spirit. Nueva York: Simon y Schuster.

Unesco (2005). Toward Knowledge Societies. Unesco World Report. Recuperado el 12 de febrero de 2013 de: http://www.unesco.org/ulis/cgi-bin/ ulis.pl? catno $=141843 \&$ set $=514$ E3B1B_1_433y$\mathrm{gp}=1 \mathrm{ylin}=1 \mathrm{yll}=1$

Van Dijk, J. (2006). The network society: Social aspects of new media. 2a. ed. Thousand Oaks, CA: Sage.

Vygotsky, L.S. (1978). Mind in Society: The Development of Higher Psychological Processes. Cambridge, MA: Harvard University Press.

Warschauer, M. (2011). Learning in the Cloud: How (and Why) to Transform Schools with Digital Media. Nueva York, NY: Teachers College Press

Watulak, S.L. y Kinzer, C.K. (2012). Beyond Technology Skills: Toward a Framework for Critical Digital Literacies in Pre-Service Technology Education. En: J. Ávila, y Z. Pandya (eds.). Critical Digital Literacies as Social Praxis: Intersections and Challenges (pp. 127-153). Nueva York: Peter Lang.

Wenglinsky, H. (1998). Does It Compute? The Relationship Between Educational Technology and Student Achievement in Mathematics. Princeton, NJ: Educational Testing Service.

Zhao, Y.; Pugh, K.; Sheldon, S. y Byers, J.L. (2002). Conditions for Classroom Technology Innovations. Teachers College Record, 104, 482-515.

\section{(C) $(1) \Theta \Theta$}

